\title{
A Two Stage Approach with ICA and Double Density Wavelet Transform for Artifacts Removal in Multichannel EEG Signals
}

\author{
Vandana Roy ${ }^{1}$ and Shailja Shukla ${ }^{2}$ \\ ${ }^{1}$ Department of Electronics \& Communication, \\ GGITS, Jabalpur, M.P., 482005, India \\ ${ }^{2}$ Professor and Head of Department of Computer Science Engineering, JEC, \\ Jabalpur, MP, 482011, India \\ vandana.roy20@gmail.com,shailja270@gmail.com
}

\begin{abstract}
Presence of artifacts in electroencephalographs (EEG) is major hurdles for the precise analysis of spectral behavior. For suppression of ocular artifact in EEG this paper proposes a component based Independent Component Analysis (ICA) model. It involves the generating a set of individual components of given signal followed by rejection of unwanted artifacts. Further this paper presents a novel method with combination of ICA, information sharing and double density wavelet transform to reject the artifacts from the signal. The Independent Component Analysis (ICA) here is used to segment artifact peaks in the signal. Then the Discrete Wavelet Transform is applied for multi-level transfer of signal data until the reception of significant result. The Wavelet ICA suppression not only removes artifacts but also preserves the spectral and coherence properties of brain signals.
\end{abstract}

Keywords: Artifacts, EEG, Double Density Wavelet, ICA

\section{Introduction}

EEG artifacts are the recorded signals that are non-cerebral in origin. The EEG signals are divided into one of two categories depending on their origin: physiological artifacts and non-physiological artifacts [1]. The amplitude a signal is subjected to the activity of cortical signals taken into account. The general categories of artifacts that are entertained includes: ECG, EMG, EOG, conscious or unconscious body activity, electrodes etc. The details of the effect of these categories are given in [2]. Artifacts are ubiquitous i.e. they are present in every EEG tracing. Artifacts on being obscure the EEG activity and render the EEG uninterruptable. Williams et al. [16] said, "Artifacts can mimic almost any kind of cerebral activity and lead to serious interpretation."

The artifact removal methods focus on dismantling the EEG epochs that lies outside the range of threshold values. Most of these methods are statistical in nature and does not consider any portion of signal outside their range. An example of such strategies such as regression offline method [1] and Fourier transform [2] leads in loss of data.

Ocular artifacts are significant observation in EEG [3, 4, 5, 6, 7], It involves skilled resource to remove manually or semi-automated method which influences majority of meaningful data loss for subsequent analysis. The presence of eye blink signals in neural signals is evident and bidirectional in nature. For separation of EOG signals, regression methods are employed for differentiating EOG from EEG.

A component based automated separator of artifacts is required to overcome this issue based on linear decomposition of signals into source components. The components give individual nature of information, where artifacts information combines into separate sources and reconstruction of signals without this source are claimed as artifact free information. P. Levan [19] Hyvarinen A and Oja E [20] on a 30s window size applied 
FastICA Algorithm 8using EEGLAB platform. They developed an automated system for artifact removal based on ICA and Bayesian Classification. Hemant K. Sawant and Zahra Jalali [18] analyzed EEG waves by DWT for frequency domain analysis. P. Senthil Kumar et al. [21] presented a statistical method based on wavelet transform to mimic ocular artifacts in EEG. Haslaile. Abdullah proposed wavelet based image processing technique at various window size. 1-D double density and 1-D double density complex were tested at window size of 10s, 30s, 60s and 300s for EEG signals.

\section{ICA based Method}

The individual signals from a multichannel source can be breakdown into their respective forms using the blind source separation method called independent component analysis.

Let us consider multichannel EEG analysis; A is the input matrix rows of multichannel with different electrodes arrangement, with assumption that there is no delay of communication between electrodes. $\mathrm{Z}$ is the output matrix consisting independent components, which is given by

$$
Z=W * A
$$

Artifact free EEG is

$$
X^{n}=(W) \text { inverse } * Z^{\prime}
$$

In $\mathrm{Z}$ row those represent artefacts are set to be zero.

Kurtosis is sourced for measure of non-gaussianity in ICA. The implemented FastICA Implementation [8] is available at [22]. FastICA is currently one of the most refined ICA techniques available [9]; this algorithm has many advantages over alternative ICA implementation. The algorithm uses an efficient fixed point iteration method that looks for local extreme points in kurtosis approach for linear arrangement of resultant points.

With this technique spectral enhancement can be achieved but at the same time it very difficult to estimate the variances of Independent components [10]. To make more precise analysis in time and frequency further wavelet technique is adopted [15].

\section{Double Density DWT}

The wavelet technique came into existence to overcome the resolution limitations of spectral analysis of Fourier Transform and denoise the corrupted signal. Selesnick et al. [11] in his research paper considered dual density wavelet transform as the substitution to Daubechies wavelet and configured filters at minimal length to suit polynomial aspects in an oversampled framework. Due to doubling in number of wavelets at every step, the proposed algorithm has larger computation time compared with DWT. The algorithm upgraded in many forms from 1-D, 2-D up to double density complex [12]. The implementation of DDWT is powered with oversampled analysis along with synthesis filters to study discrete signals (see figure 2). The given wavelets consists a frequency response (see figure 1) for $h^{0}, h^{1}, h^{2}$ filters (filter length of seven, seven and five respectively). The first filter is low pass and rests of two are high pass (having equal response in magnitude). The synthesis and analysis banks incorporate its low pass segment for oversampling and the repetitive process creates an oversampled filter bank. This architecture can be seen as the substitution to multi resolution analysis having $\varnothing$ as the scaling function for $\varphi^{1}{ }_{v} \varphi^{2}$ wavelets.

The double density DWT is applying the oversampled filter bank for a low pass sub band signal $c(n)$. In this paper the double density algorithm is used along with the filter banks for sampling purpose. 


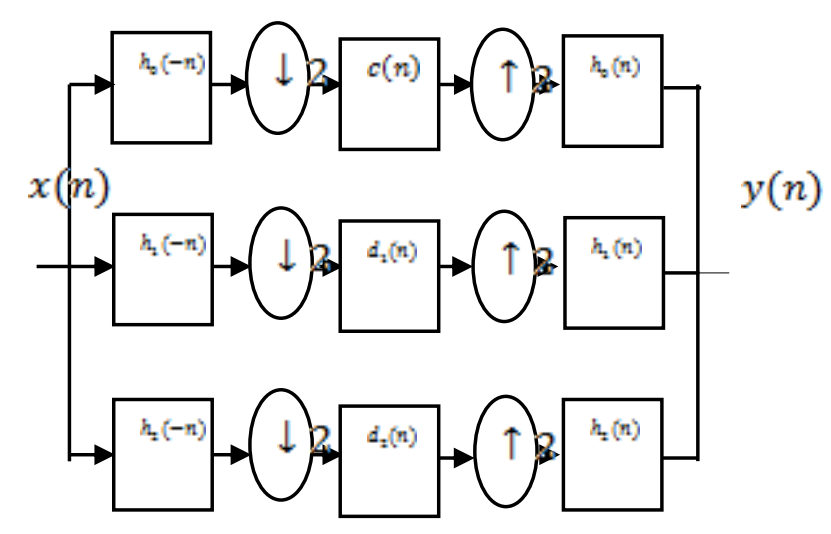

Figure 1. Frequency Responses for the Analysis-bank Filters

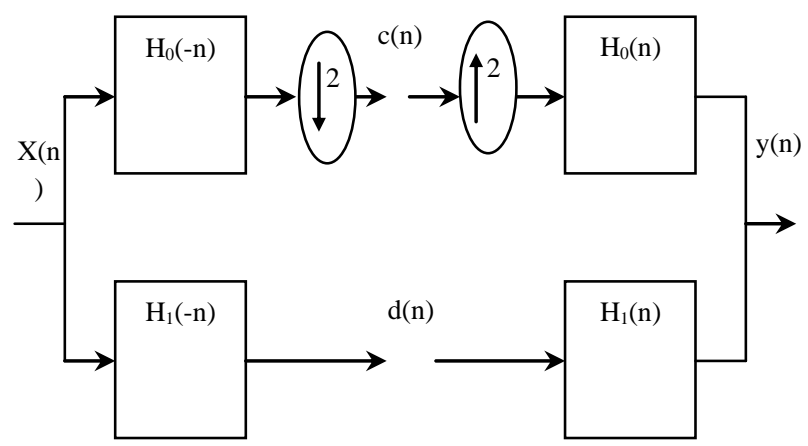

Figure 2. Block Representation of Filter Bank on which Realizable Filters are Inefficient

To create a combination of DD-DWT along with FIR filters, an oversampled filter bank (figure 2) with low and high pass filters is used.

The relation among $Y(z)$ and $X(z)$ are explored that results in efficient reconstruction conditions

$Y(z)=\frac{1}{2}\left[H_{0}(z) H_{0}\left(\frac{1}{z}\right)+H_{1}(z) H_{1}\left(\frac{1}{z}\right)+H_{2}(z) H_{2}\left(\frac{1}{z}\right)\right] X(z)+$
$=\frac{1}{2}\left[H_{0}(z) H_{0}\left(-\frac{1}{z}\right)+H_{1}(z) H_{1}\left(-\frac{1}{z}\right)+H_{2}(z) H_{2}\left(-\frac{1}{z}\right)\right] X(-z)$

The filter bank employed to structure the double-density discreet wavelet transform resembles the wavelet frame with $\phi(t)$ as scaling function and $\psi_{1}(t)$ and $\psi_{2}(t)$ wavelets. According to the documents of dyadic wavelet space

$V_{j}=\operatorname{Span}_{n \in Z}\left\{\phi\left(2^{j} t-n\right)\right\}$

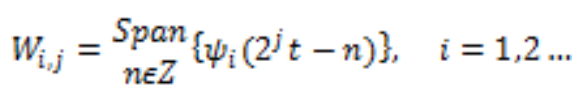

The single wavelet $\psi$ and scaling function $\phi$ defines the structure of a dyadic wavelet. The additional wavelet in equation 4 avoids any possibility of incompleteness. In terms of multi-resolution framework, the nesting of $V_{0} \subset V_{1}, W_{1,0} \subset V_{1}, W_{2,0} \subset V_{1}$ can be demanded. Hence, the wavelets and scaling function satisfies the dilation equations

$$
\begin{aligned}
\varnothing(t)=\sqrt{2} \sum_{n} h_{0}(n) \emptyset(2 t-n) & \\
\psi_{i}(t) & =\sqrt{2} \sum_{n} h_{\mathrm{i}}(n) \emptyset(2 t-n) \quad i=1,2 \ldots
\end{aligned}
$$


$h_{0}(n) h_{1}(n)$ and $h_{2}(n)$ from III section illustrates about wavelets $\psi_{1}(t), \psi_{2}(t)$ and scaling function of equation $\varnothing(t) 5$.

\section{Proposed Method}

A proposed novel technique used both the wavelet transfer\& ICA for removal of artifacts from the given signal. The DDWT (double density wavelet-transform) is inefficient in minimizing overlapping artifacts in spectral domain. By considering the assumption the no of source is equal to no of sensor; the DDWICA (double density wavelet transform ICA) can be used for overlap artifacts removal.

In DDWICA single channels recording are used because wavelet decomposition creates multi-dimensional data for single measurements [4, 13]. The resulting waveletresolution is selected based on hard and soft thresholding.

The reference from Markov model of coefficients illustrates the significant amplitude values of artifacts in time domain spectrum. The coefficients of neural signals possess lower energy and wider spectrum.

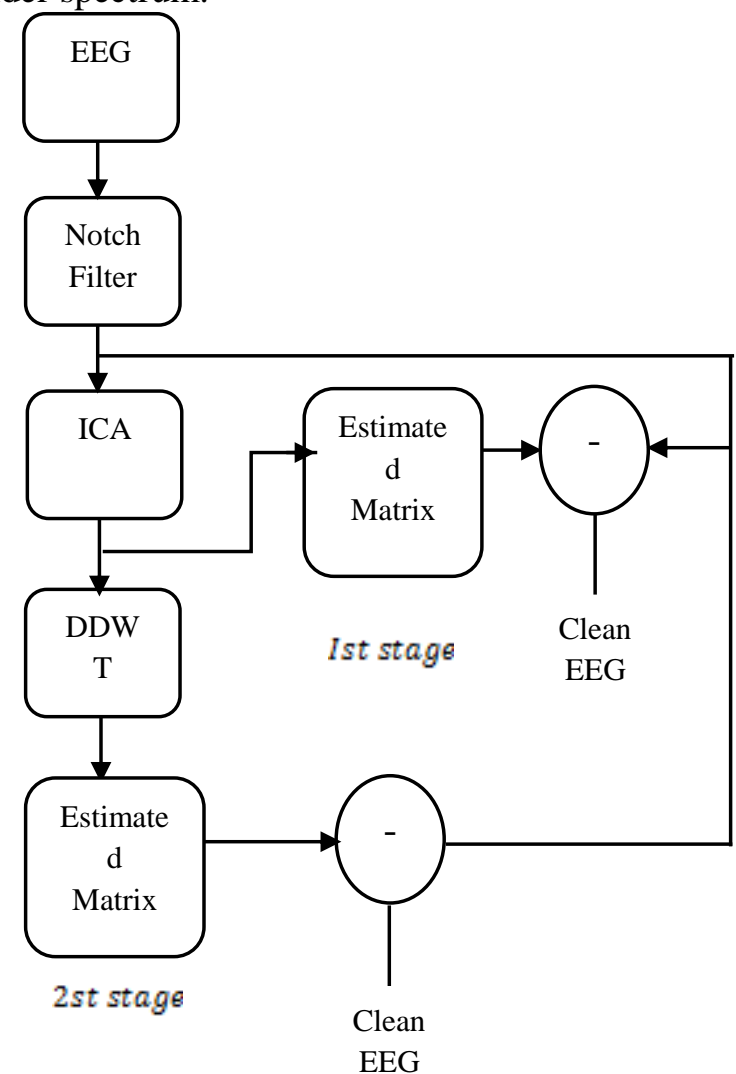

Figure 3. Two Stage Artifacts Suppression using ICA and Double Density Wavelet Transform

Let $\mathrm{D}$ is the data with combination of $a(t)$ high amplitude artifacts and $n(t)$ small and homogenously spaced low energy.

$$
D(t)=a(t)+n(t)
$$

Independent component

$\omega^{s}(d, b)=\frac{1}{\sqrt{\alpha}} \int S_{1}(t) \sqrt{2} \sum_{n} h_{\mathrm{i}}(n) \varnothing(2 t-n) \quad i=1,2 . \cdot d t$ 
Where $\omega^{s}$ is the wavelet

Representation of $S_{1}(t) \sqrt{2} \sum_{n} h_{\mathrm{i}}(n) \emptyset(2 t-n)_{s} i=1,2 \ldots$ is the double density wavelet coefficient

From $6 \& 7$ can be written as

$$
\omega^{a}(d, b)=\omega^{a}(d, b)+\omega^{n}(d, b)
$$

Where, $\omega^{a}(d, b) \& \omega^{n}\left(d_{s} b\right)$ are the wavelet coefficient of artifacts $\&$ neural points.

$k=\sqrt{2} \log N \sigma$ (Threshold)

$\mathrm{N}=$ Length of data segments

$\sigma=$ Variance which act as regulator for artifacts removal

After that filtration again the inverse transform of the double density wavelet coefficient is performed for reconstruction of original neural components. Clean EEG are obtained by subtracting the filter out put to reconstructed signal.

\section{Experimental Setup}

\section{Test Inputs}

The database for EEG signals was collected from healthy subjects [14] practicing the standard parameters of formulation. The information about process and subjects is collected from [14] and same is presented here. The Electro-Cap System is employed in system with 19 scalp electrodes placed according to the International 10-20 System. The signals were digitized at a rate of $256 \mathrm{~Hz}$ and further filtered (notch filter at $50 \mathrm{~Hz}$, and band pass filter $4-45 \mathrm{~Hz}$ ).

The second source of data is http://www.appliedneuroscience.com/Cal-2Hz-increment$100 \mathrm{uVp}$-p.tdt.

The third data is available athttp://www.vis.caltech.edu/ rodri/data.htm. The third source of data is 30 multi-unit recording of a patient with epileptic disease taken at ItzahakFrieds lab at UCLA.

\section{Performance Evaluation Parameters}

Mean Square Error $=\frac{1}{n} \sum_{i=1}^{n}\left(\hat{Y}_{i}-Y_{i}\right)^{2}$

Where,

$\hat{Y}=$ Vectors of $\mathrm{n}$ predictions

$Y=$ Vectors of True Values

$\mathrm{n}=$ Total number of samples

Power spectrum density:

The supplemented readings of Electro encephalography signals are sourced by Gaussian noise. The obtained variation in signals in reference to theoretical model leads to restricted access in accuracy of power spectrum of signals (Table 1).

$P_{x z}(w)=P_{r y}(w)+\Delta P(w)$

Where,

$P_{r y}=$ Reference Power Spectrum of Artifact supressed signal

$P_{x y}=$ Power Spectrum of ICA modified Signal

$P=$ Distortion Spectrum by side effects of methods (Should be 0 ideally)

$$
\Delta P_{j}(w)=-m_{j 1}^{2} P_{n n}(w)
$$

Here, 


\section{$m_{j 1}$ Denotes weight from Matrix M}

$P_{n n}$ Component of brain signals in ICA

Equation 11 represents the minimization of ICA-EEG based on spectral function $\left(P_{n n}\right)$ with factors $m_{j 1}^{2}$. As there exists direct relationship among decrease in $j$ and $m_{j 1}^{2}$, front end experience high distorted signals of spectrum. DDWICA along with reduction of residual EEG signals in artifact components simultaneously minimize $P_{n n}$ on a serious note that leads to improved approximated value of pure EEG power spectrum.

Quantifying the distortion degree that were present in readings cause of artifact minimization scheme, the mean power density to average ten epochs is calculated for conversion in decibels.

\section{Suppression of artifacts by ICA and Double density wavelet ICA methods}

Consider an example for minimization of artifact signals occurred due to blinking and heart poundings. The nature of blink artifacts in EEG signals has high amplitude while the heartbeat artifacts are recognized for their short signals train with lower amplitude. ICA algorithm classifies artifacts and brain signals into nineteen separate components independent in nature. The foremost components of independent analysis are healthy in nature and are seen at front end. These components clashes with blink artifacts with characteristics like profound low amplitude signal pulse and scalp topology exhibits neat frame at front end [4]. With this knowledge of components, first component is acknowledged as responsible candidate for ocular artifacts. In the same manner, the components at fourth stage are pulses of rhythmic heart beat noise.

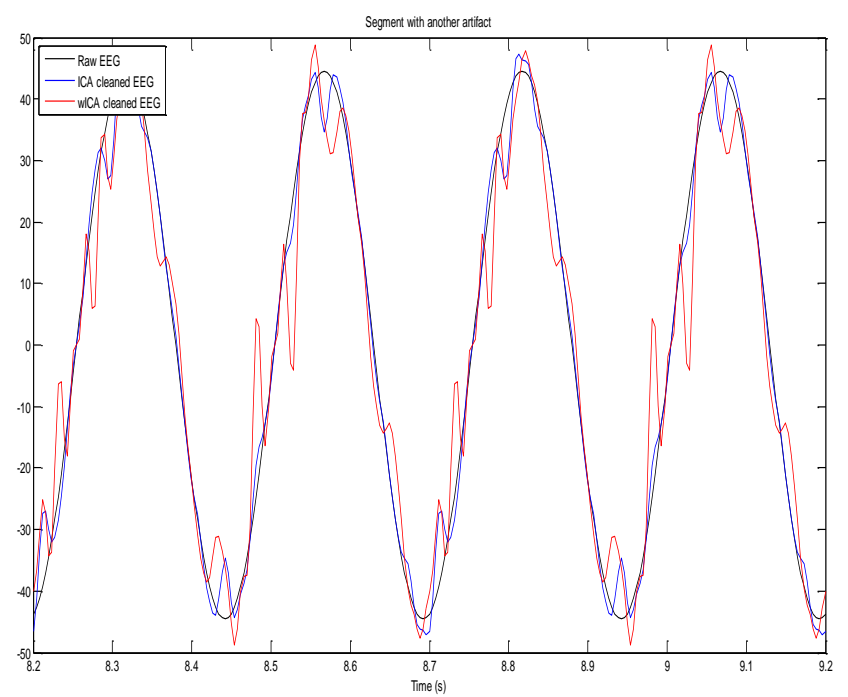

Figure 4. Comparative Analysis of $2 \mathrm{~Hz}$-increment-100uVp EEG Data with 19Channel 

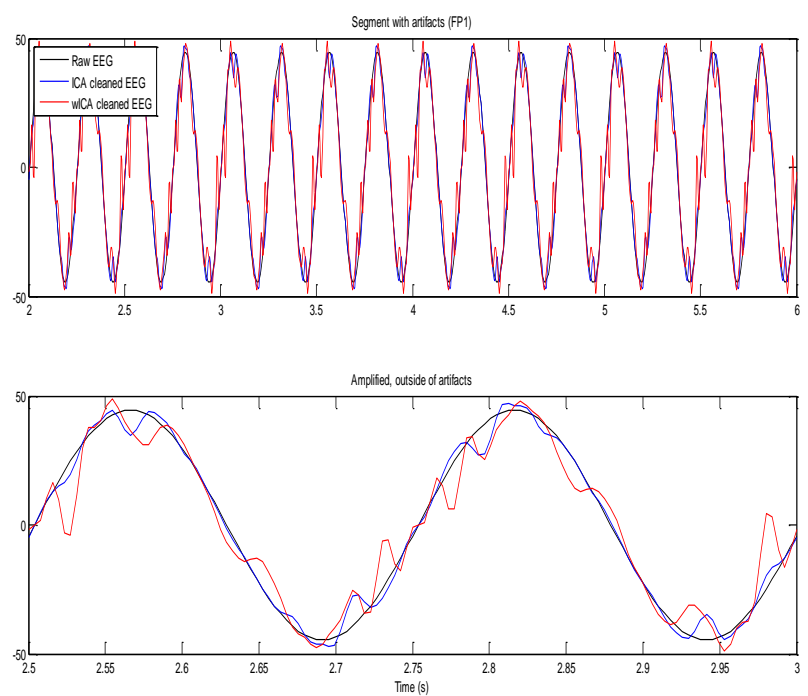

Figure 5. Comparative Analysis of 2Hz-increment-100uVp EEG Data with FP1 Channel

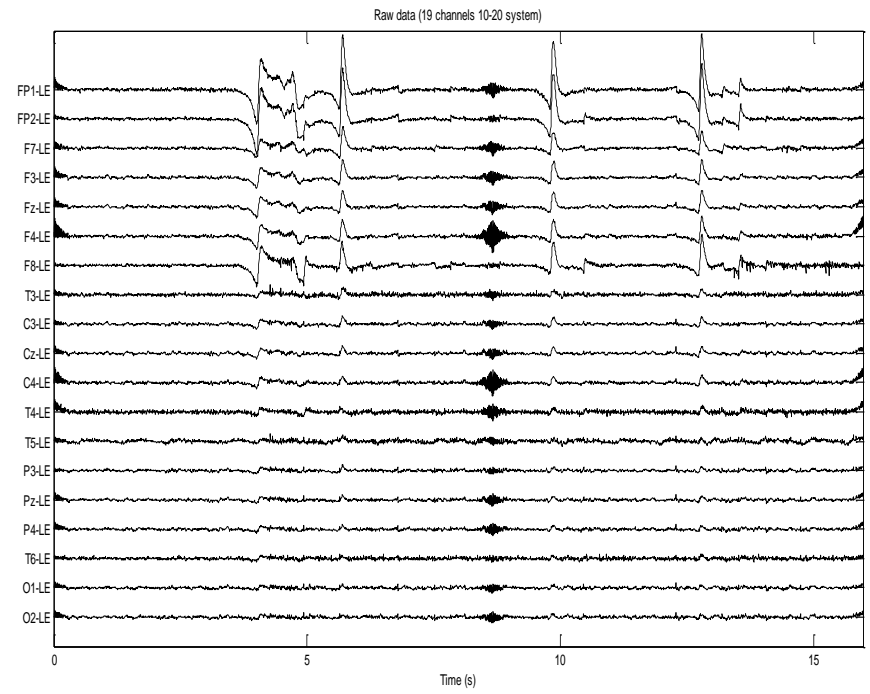

Figure 7. Plot of Raw EEG data of 19-channel Form ECI Electro-Cap System with Respect to Time 


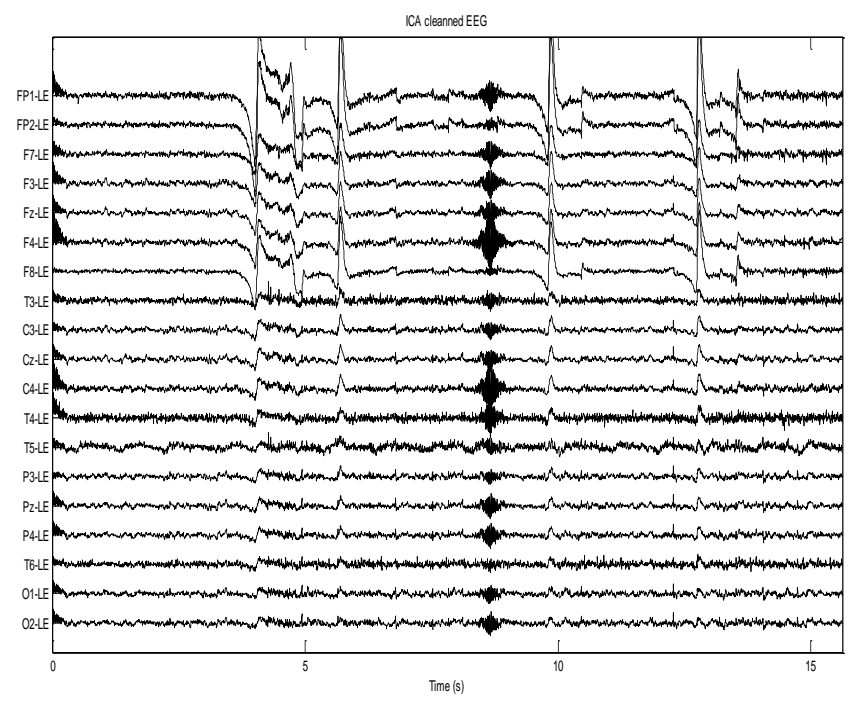

Figure 8. ICA Cleaned Data of 19 Channel form ECI Electro-Cap System with Respect to Time

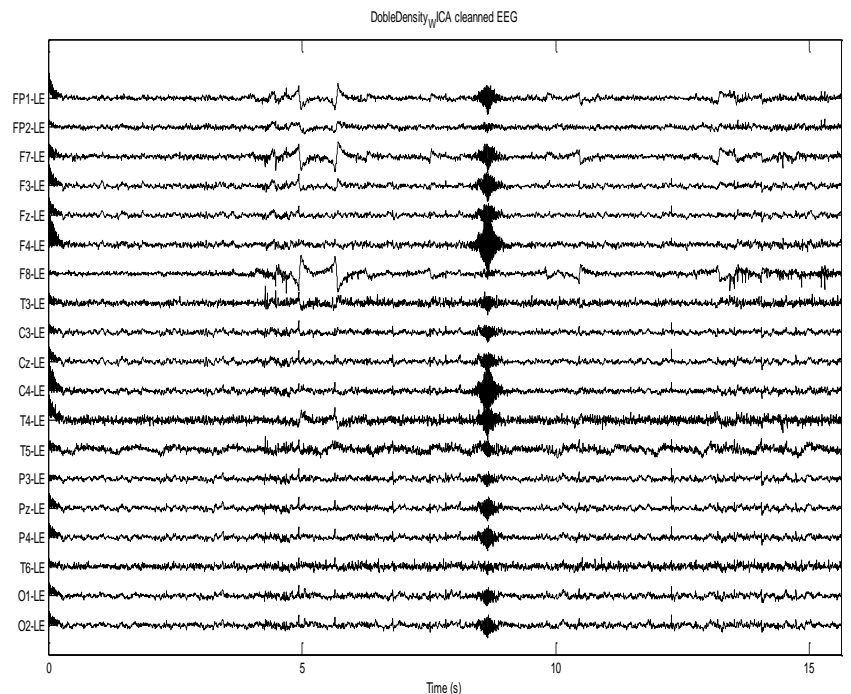

Figure 9. Double Density Wavelet Clean Data EEG Data of 19-channel Form ECI Electro-Cap System with Respect to Time 


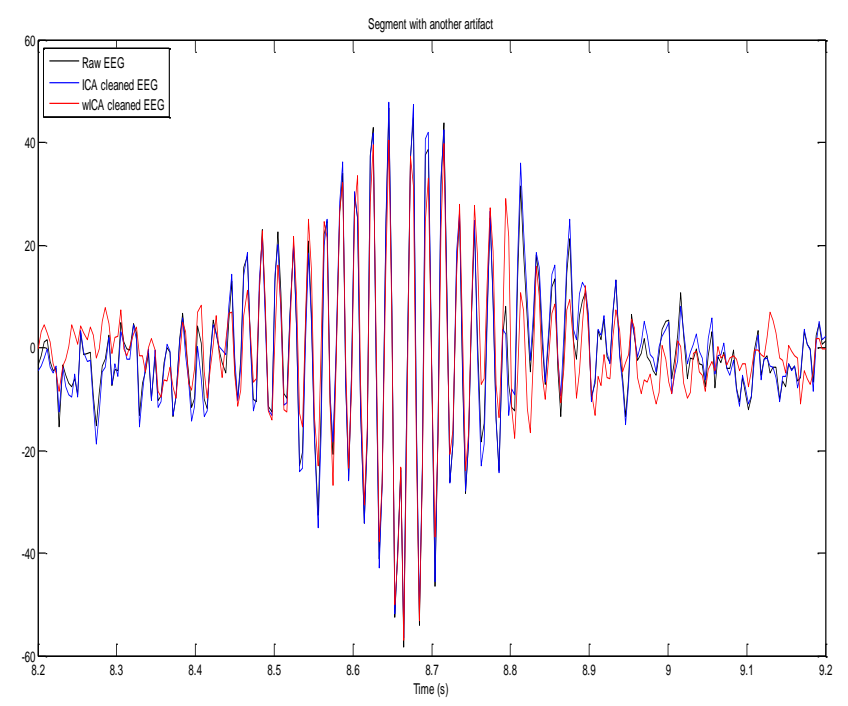

Figure 10. Comparative Analysis of Two-stage EEG Filtering with ICA and Double Density Wavelet for 19-channel form ECI Electro-Cap System
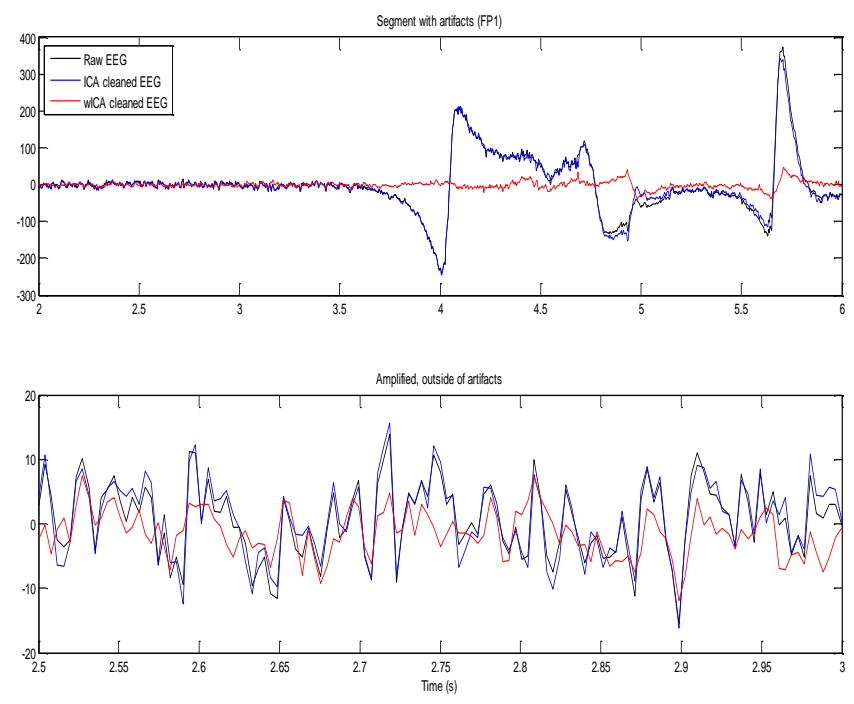

Figure 11. Comparative Analysis of Two-stage EEG Filtering with ICA and Double Density Wavelet on FP1 Channel the ECI Electro-Cap System 


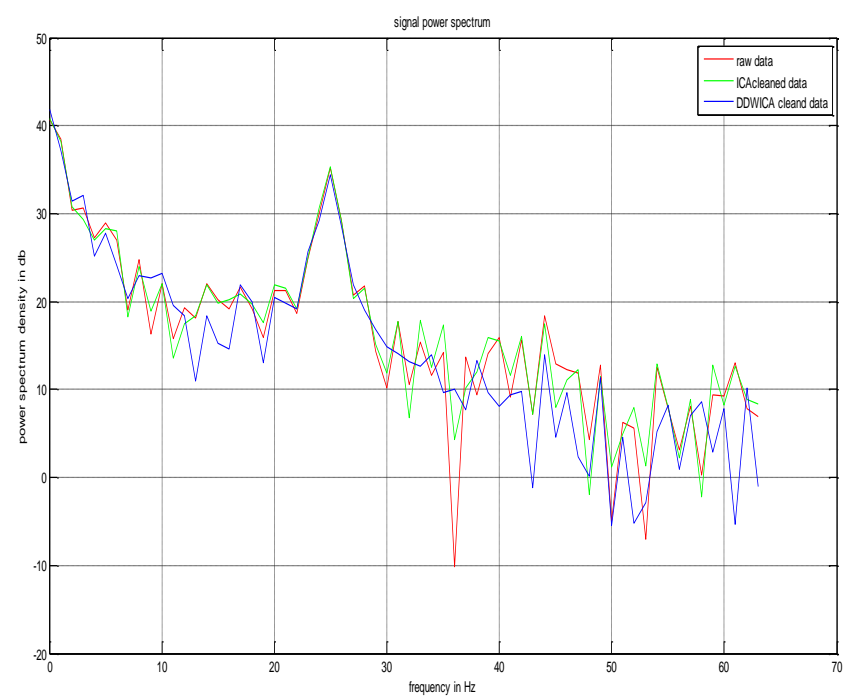

Figure 12. A Comparative Analysis of Source Signals and Filtered Signal by ICA and DDWICA in Terms of PSD is Shown for 19 Channel form ECI Electro-Cap System at $256 \mathrm{~Hz}$

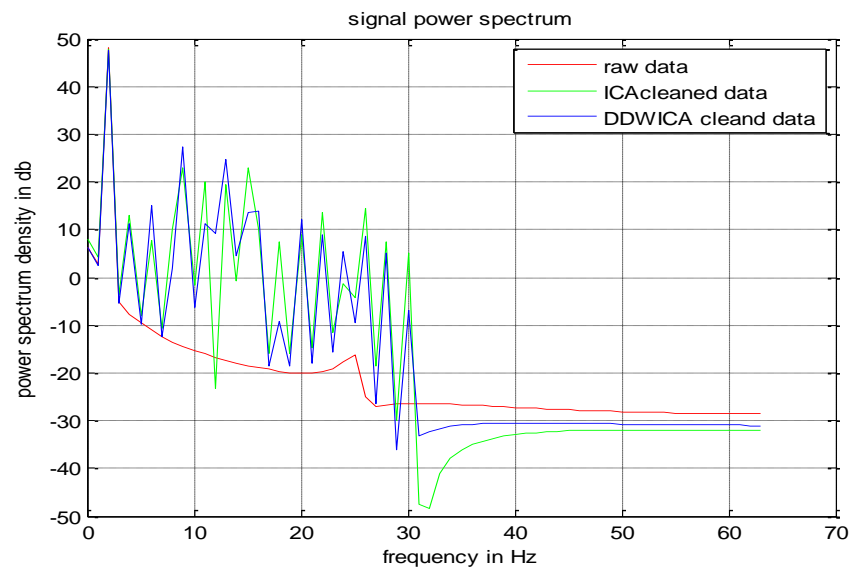

Figure13. A Comparative Analysis of Source Signals and Filtered Signal by ICA and DDWICA in Terms of PSD is Shown for $2 \mathrm{~Hz}$-increment-100uVp EEG Data with 19-channel 


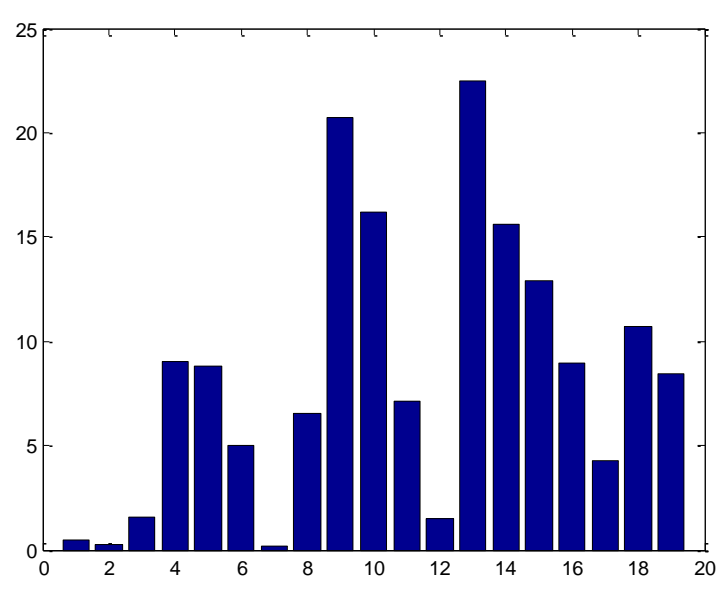

Figure 12. Statistical Representation of Mean Square Error in ICA

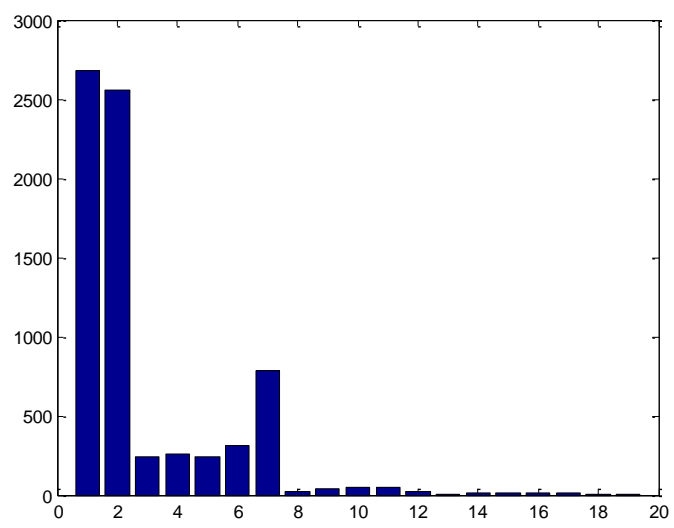

Figure 13. Statistical Representation of Mean Square Error for Two Stage Double Density Wavelet \& ICA

Table 3. MSE (Mean Square Error)

\begin{tabular}{|l|c|c|c|c|c|}
\hline $\begin{array}{c}\text { channe } \\
\text { ls }\end{array}$ & ICA & $\begin{array}{c}\text { DWTIC } \\
\mathbf{A}\end{array}$ & $\begin{array}{c}\text { channel } \\
\mathbf{s}\end{array}$ & ICA & $\begin{array}{c}\text { DWTIC } \\
\mathbf{A}\end{array}$ \\
\hline FP1-LE & 11.797 & 22.676 & FP1-LE & 0.457 & $\begin{array}{c}2675.62 \\
7\end{array}$ \\
\hline FP2-LE & 3.996 & 9.609 & FP2-LE & 0.265 & $\begin{array}{c}2555.61 \\
9\end{array}$ \\
\hline F7-LE & 2.626 & 3.816 & F7-LE & 1.599 & 245.40 \\
\hline F3-LE & 4.387 & 9.026 & F3-LE & 9.007 & 259.73 \\
\hline F2-LE & 0.695 & 0.767 & F2-LE & 8.835 & 243.69 \\
\hline F4-LE & 0.006 & 1.152 & F4-LE & 5.008 & 312.984 \\
\hline
\end{tabular}




\begin{tabular}{|c|c|c|c|c|c|}
\hline F8-LE & 0.220 & 0.281 & F8-LE & 0.216 & 782.12 \\
\hline T3-LE & 2.238 & 8.324 & T3-LE & 6.578 & 22.058 \\
\hline C3-LE & 2.065 & 3.116 & C3-LE & 20.729 & 42.848 \\
\hline C2-LE & 2.378 & 7.803 & C2-LE & 16.212 & 45.919 \\
\hline C4-LE & 10.372 & 21.377 & C4-LE & 7.139 & 47.451 \\
\hline T4-LE & 0.012 & 0.1198 & T4-LE & 1.496 & 22.789 \\
\hline T5-LE & 10.809 & 16.335 & T5-LE & 22.458 & 8.462 \\
\hline P3-LE & 3.088 & 6.553 & P3-LE & 15.589 & 15.555 \\
\hline P2-LE & 4.747 & 6.707 & P2-LE & 12.932 & 16.072 \\
\hline P4-LE & $\begin{array}{c}402.01 \\
8\end{array}$ & 776.09 & P4-LE & 8.985 & 16.000 \\
\hline T6-LE & $\begin{array}{c}193.91 \\
8\end{array}$ & 407.836 & T6-LE & 4.240 & 10.625 \\
\hline O1-LE & $\begin{array}{c}222.47 \\
1\end{array}$ & 462.537 & O1-LE & 10.681 & 6.758 \\
\hline O2-LE & 40.239 & 79.574 & O2-LE & 8.408 & 6.496 \\
\hline
\end{tabular}
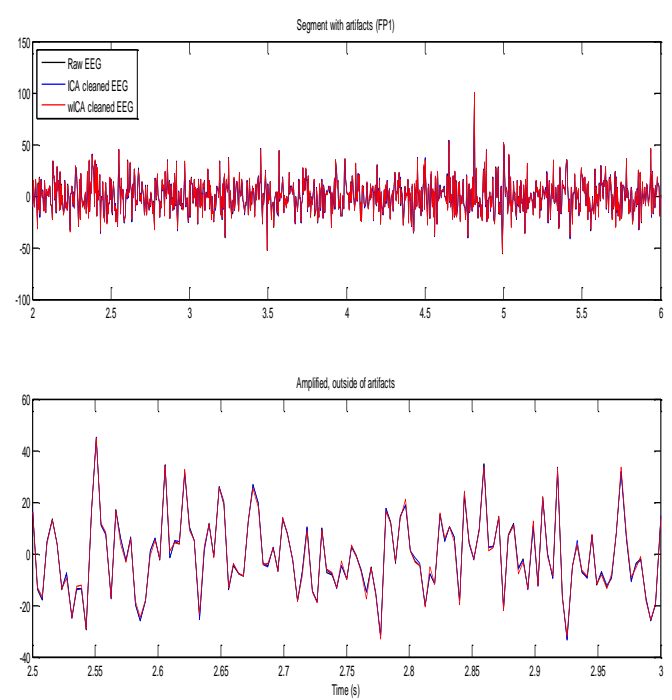

Figure 14. Example is a 30 Multi Unit Recording in Medical Temporal Lobe of an Epileptic Patient from ItzhakFrieds Lab 


\section{Result Analysis}

Figure 7 shows the Plot of Raw EEG data of 19-channel form ECI Electro-Cap System with respect to time. All recording channels are taken on universal reference electrode at LE. All signals were sampled at $256 \mathrm{~Hz}$ frequency cut off frequency $40 \mathrm{~Hz}$. Figure 8 shows ICA cleaned data of 19 channel form ECI Electro-Cap System with respect to time. Here channel 2 was with ocular artifacts which further suppressed by ICA and channel F8 represents the Heart beat artifacts. Figure 9 shows Double density wavelet clean data EEG data of 19-channel form ECI Electro-Cap System with respect to time. Here channel 2 was with ocular artifacts which further suppressed by ICA and channel F8 represents the Heart beat artifacts. Result shows better filtration of ocular artifacts then ICA and fewer fluctuations. Figure 10 shows comparative analysis of two-stage EEG filtering with ICA and Double density wavelet for 19-channel form ECI Electro-Cap System. Figure 11 shows comparative analysis of two-stage EEG filtering with ICA and Double density wavelet on FP1 Channel the ECI Electro-Cap System having 19 scalp electrodes arranged in International 10-20 format. Above graphs represents the FP1 channel with common reference LE and shows better stability and artifacts free with DDWICA as compared with ICA. Figure 12 shows comparative analysis of source signals and filtered signal by ICA and DDWICA in terms of PSD is shown for 19 channel form ECI Electro-Cap System at $256 \mathrm{~Hz}$. The DDWICA-transformed signal resembles with original signal in terms of PSD for certain band, ahead of that $40 \mathrm{~Hz}$ ICA reconstructed signal replicate better then DDWICA. Figure13 shows comparative analysis of source signals and filtered signal by ICA and DDWICA in terms of PSD is shown for $2 \mathrm{~Hz}-$ increment-100uVp EEG data with 19-channel The DDWICA-transform reconstructed signal possess better PSD with $30 \mathrm{~Hz}$ than ICA, it shows better correlation then ICA with raw EEG signals.

\section{Conclusion}

In this paper the problem of artifacts removal for EEG signal has been addressed. A two stage ICA and double density wavelet transform has been integrated for automatic artifacts removal. Graphical analysis shows that double density WICA outperforms better then ICA. Mean square error is calculated to evaluate the performance of removal on 19 channel separately. A further study has been focus on quantitative analysis of how much distortion or information loss is because of automatic artifact removal with spectral power density. The statistics shows that two-stage preforms better then ICA based method.

\section{References}

[1] http://cognitrn.psych.indiana.edu/busey/eegseminar/pdfs/EEGPrimer Ch6.pdf

[2] Iván Manuel Benito Núñez, "EEG Artifact Detection”, Project Report, Department of Cybernetics Czech Technical University, Prague, (2010).

[3] A. Flexer, "Using ICA for removal of ocular artifacts in EEG recorded from blind subjects", Neural Networks, vol. 18, no. 7, (2005), pp 998-1005.

[4] M. A. Klados, "REG-ICA: A hybrid methodology combining Blind Source Separation and regression techniques for the rejection of ocular artifacts", Biomedical Signal Processing and Control , vol. 6, no. 3, (2011), pp 291-300.

[5] J. P. Lindsen and J. Bhattacharya, "Correction of blink artifacts using independent component analysis and empirical mode decomposition", Psychophysiology , vol. 47, no. 5, (2010), pp. 955-960.

[6] J. Escudero, "Quantitative evaluation of artifact removal in real magneto encephalogram signals with blind source separation", Annals of biomedical engineering, vol. 39, no. 8, (2011), pp. 2274-2286.

[7] A. Flexer, H. Bauer, J. Pripfl, G. Dorffner, "Using ICA for removal of ocular artifacts in EEG recorded from blind subjects”, Neural Networks, vol. 18, no. 7, (2005), pp. 998-1005.

[8] A. Hyv"arinen, P. Pajunen, "Nonlinear independent component analysis: existence and uniqueness results", Neural Networks, vol. 12, no. 3, pp 209-219, (1999). 
[9] J.-C. Chao and S. C. Douglas, "A robust complex FastICA algorithm using the huber M-estimator cost function", In Independent Component Analysis and Signal Separation, Springer Berlin Heidelberg, vol. 4666, (2007), pp 152-160.

[10] S. Arora, "Provable ICA with unknown Gaussian noise, and implications for Gaussian mixtures and autoencoders", Advances in Neural Information Processing Systems, vol. 1206, no. 5349, (2012), pp. 2375-2383.

[11] I. W. Selesnick, "The double density DWT", Wavelets in Signal and Image Analysis, Springer Netherlands, vol. 19, (2001), pp. 39-66.

[12] S. Chitchian, "Retinal optical coherence tomography image enhancement via shrinkage denoising using double-density dual-tree complex wavelet transform", Journal of biomedical optics, vol. 17, no. 11, , (2012), pp. 116009-116009.

[13] G. Brown, S. Yamada, T. Sejnowski, "Independent component analysis at the neural cocktail party", Trends Neuroscience, vol. 24, no. 1, (2001), pp. 54-63.

[14] G. Tan, "Meta-analysis of EEG biofeedback in treating epilepsy", Clinical EEG and Neuroscience, vol. 40, no. 3, (2009), pp. 173-179.

[15] P. Kumar, Senthil, "Removal of Ocular Artifacts in the EEG through Wavelet Transform without using an EOG Reference Channel", Int. J. Open Problems Compt. Math, vol. 1, no. 3, (2008), pp 188-200.

[16] G. W. Williams, H. O. Luders, A. Brickner, M. Goormastic, D. W. Klass, "Inter Observer Variability in EEG Interpretation", Neurology, vol. 35, no. 12, (1985), pp. 1713-19.

[17] A. Haslaile and C. Dean, "Double Density Wavelet For EEG Signal Denoising" 2nd International Conference On Machine Learning And Computer Science (IMLCS'2013) August 25-26, (2013); Kuala Lumpur, Malaysia.

[18] H. K. Sawant and Z. Jalali, "Detection And Classification Of EEG Waves", Oriental Journal Of Computer Science \& Technology, vol. 3, no. 1, (2010), pp. 207-213.

[19] A. Hyvarinen, E. Oja, "A Fast fixed-Point Algorithm For Independent Component Analysis", Neural Computer, vol. 9, no. 7, (1997), pp. 1483-1492.

[20] P. Levan, E. Urrestarrazu, and J. Gotman, "A System For Automatic Artifact Removal In Ictal Scalp EEG Based On Independent Component Analysis And Bayesian Classification”, Clinical Neurophysiology, vol 117, no. 4, pp. 912- 927, (2006).

[21] P. Senthil Kumar, R. Arumuganathan, K. Sivakumar, And C. Vimal, "Removal Of Ocular Artifacts In The EEG Through Wavelet Transform Without Using An EOG Reference Channel", Int. J. Open Problems Compt. Math., vol. 1, no. 3, pp. 188-200, (2008).

[22] http://www.cis.hut.fi/projects/ica/fastica 